\title{
Unexpected Survival of Neurons of Origin of the Pyramidal Tract after Spinal Cord Injury
}

\author{
Jessica L. Nielson, ${ }^{1,2}$ Ilse Sears-Kraxberger, ${ }^{1}$ Melissa K. Strong, ${ }^{1,2}$ Jamie K. Wong, ${ }^{1,3}$ Rafer Willenberg, ${ }^{1,2}$ \\ and Oswald Steward ${ }^{1,2,3,4}$ \\ ${ }^{1}$ Reeve-Irvine Research Center, Departments of ${ }^{2}$ Anatomy and Neurobiology, ${ }^{3}$ Neurobiology and Behavior, and ${ }^{4}$ Neurosurgery, University of California, \\ Irvine, Irvine, California 92697
}

There is continuing controversy about whether the cells of origin of the corticospinal tract (CST) undergo retrograde cell death after spinal cord injury (SCI). All previous attempts to assess this have used imaging and/or histological techniques to assess upper motoneurons in the cerebral cortex. Here, we address the question in a novel way by assessing Wallerian degeneration and axon numbers in the medullary pyramid of Sprague Dawley rats after both acute SCI, either at cervical level 5 (C5) or thoracic level 9 (T9), and chronic SCI at T9. Our findings demonstrate that only a fraction of a percentage of the total axons in the medullary pyramid exhibit any sign of degeneration at any time after SCI-no more so than in uninjured control rats. Moreover, design-based counts of myelinated axons revealed no decrease in axon number in the medullary pyramid after SCI, regardless of injury level, severity, or time after injury. Spinal cord-injured rats had fewer myelinated axons in the medullary pyramid at 1 year after injury than aged matched controls, suggesting that injury may affect ongoing myelination of axons during aging. We conclude that SCI does not cause death of the CST cell bodies in the cortex; therefore, therapeutic strategies aimed at promoting axon regeneration of the CST in the spinal cord do not require a separate intervention to prevent retrograde degeneration of upper motoneurons in the cortex.

\section{Introduction}

Before the advent of retrograde tracing, cells of origin for fiber tracts were discovered by cutting axons and looking for cells that displayed signs of retrograde degeneration, specifically chromatolysis (Nissl, 1892). The cells of origin of the corticospinal tract (CST) were first identified in this way (Holmes and May, 1909), and since then, there has been continuing debate about whether corticospinal neurons die as a result of axotomy. Within the context of developing therapies to promote axon regeneration and recovery after spinal cord injury (SCI), this is a crucial question that needs a definitive answer. Many therapies currently being explored aim to enhance axon regeneration at the lesion site (Thuret et al., 2006). Such therapies require, however, that the cells of origin of CST axons in the motor cortex survive, because when a cell body dies, the axon dies as well (Carlson et al., 2000, 2001), which would make therapeutic interventions to induce axon regeneration futile. Numerous studies have addressed this question by assessing changes in the motor cortex after injury, with conclusions ranging from no cell death to extensive retrograde degeneration. Assessments have included counts of neurons in the cortex (Feringa and Vahlsing, 1985; Giehl and

Received March 16, 2010; revised July 2, 2010; accepted July 8, 2010.

This work was supported by National Institutes of Health Grant NS047718 (0.S.) and Grants RR07-227 and RR08-263 from the Roman Reed Spinal Cord Injury Research Fund of California. The colony of rats with chronic spinal cord injury was supported by private donations to the Reeve-Irvine Research Center from "Research for Cure." Thanks to Kelli Sharp for technical assistance.

Correspondence should be addressed to Dr. Oswald Steward, 1105 GNRF, 837 Health Sciences Drive, University of California, Irvine, Irvine, CA 92697. E-mail: osteward@uci.edu.

DOI:10.1523/JNEUROSCI.1433-10.2010

Copyright $\odot 2010$ the authors $\quad 0270-6474 / 10 / 3011516-13 \$ 15.00 / 0$
Tetzlaff, 1996; Hammond et al., 1999; Bonatz et al., 2000; Hains et al., 2003; Klapka et al., 2005), changes in gene expression in the cell bodies (Kost-Mikucki and Oblinger, 1991; Mikucki and Oblinger, 1991; Kost and Oblinger, 1993; Mason et al., 2003), changes in forelimb representation (Schmidlin et al., 2004, 2005), assessment of motor responses after stimulation in the cortex (Piecharka et al., 2005), shrinkage of the cell bodies (Holmes and May, 1909; Kalil and Schneider, 1975; Ganchrow and Bernstein, 1985; Merline and Kalil, 1990; Wannier et al., 2005), chromatolysis (Holmes and May, 1909; Lassek, 1942; Bodian, 1946), and activation of cell death markers (Hains et al., 2003; Lee et al., 2004). There have also been reports of no response to injury (Mason et al., 2003; Crawley et al., 2004). One of the difficulties in all of these analyses is that the assessments were performed over widespread areas of the cerebral cortex and did not involve quantitative assessment techniques that take into account changes in neuron size (i.e., unbiased sampling and counting). Based on these considerations, the present study takes a different approach-analyzing the axons of the CST in the one site that they are found in a single, definable tract, the medullary pyramid. Essentially all CST axons travel through the medullary pyramid en route to the spinal cord. If CST cell bodies die after SCI, their axons must die too, which should be detectable as Wallerian degeneration of the axons [called indirect Wallerian degeneration (van Gehuchten, 1903)] and time-dependent decreases in axon number in the medullary pyramid.

\section{Materials and Methods}

Animal use and care

All procedures were approved by the Institutional Animal Care and Use

Committee at the University of California, Irvine, in compliance with the 
National Institutes of Health guidelines. All animals used for this study were Sprague Dawley outbred rats (Harlan Laboratories), maintained on a $12 \mathrm{~h} \mathrm{light/dark} \mathrm{cycle} \mathrm{at} 25^{\circ} \mathrm{C}$. For surgery, animals were anesthetized with an intraperitoneal injection of ketamine and xylazine (100 and 10 $\mathrm{mg} / \mathrm{kg}$, respectively; Western Medical Supply). Postoperatively, animals received subcutaneous injections of $10 \mathrm{ml}$ of $0.9 \%$ saline, $0.5 \mathrm{mg} / \mathrm{kg}$ Baytril, and $0.01 \mathrm{mg} / \mathrm{kg}$ buprenorphine, and were kept warm on an isothermic pad until mobile and alert. After injury, animals received saline and Baytril for 10 consecutive days or until animals were killed for analysis, and buprenorphine was given for 7 consecutive days. For SCI animals, bladders were manually expressed twice per day for the duration of the experiments.

\section{Injury paradigms}

Thoracic vertebral level 9 dorsal funiculus lesion. To assess CST axon degeneration after axotomy at a site relatively distal to the cells of origin in the cortex, young male rats (150-175 g at time of surgery) received a dorsal funiculus (DF) lesion at thoracic vertebral level 9 (T9), using ophthalmic microscissors (World Precision Instruments; $7 \mathrm{~cm}$ long, curved $3 \mathrm{~mm}$ blades, $0.1 \mathrm{~mm}$ tips). This injury paradigm bilaterally transects the dorsal CST and has been described previously (Neumann and Woolf, 1999; Hains et al., 2003). The completeness of the injury was verified using an operating microscope; the muscles were sutured in layers, and the skin was closed with wound clips. This injury causes paralysis of the hindlimbs, and only transient bladder impairment during the first day after surgery, and did not affect normal eating, drinking, or grooming. Animals were allowed to survive for either 1 week $(n=16)$ or 4 weeks $(n=7)$ after injury.

Cervical vertebral level 5 lateral hemisection lesion. To assess axon degeneration after axotomy at a site relatively proximal to the cells of origin in the cortex, adult female rats (225-250 g at time of surgery) received a right lateral hemisection lesion $(\mathrm{Hx})$ at cervical vertebral level 5 (C5) using a Moria microknife (FST catalog \#7040A; Fine Science Tools). This injury paradigm unilaterally transects the crossed dorsal and dorsolateral and uncrossed ventral components of the CST and has been described previously (Anderson et al., 2005). The completeness of the injury was verified using an operating microscope; the muscles were sutured in layers, and the skin was closed with wound clips. This injury causes paralysis of the forearm and paw ipsilateral to the injury, with partial ipsilateral hindlimb paralysis, and transient bladder impairment for the first day after surgery. Eating and drinking were not impaired; however, grooming was predominantly with the left forelimb. Animals were allowed to survive for either 1 week $(n=8)$ or 3 weeks $(n=8)$ after injury.

Retrograde tracing. Immediately after both the T9 DF and C5 Hx injury paradigms, the majority of animals received a $0.5 \times 0.5 \times 0.5 \mathrm{~mm}$ piece of Gelfoam (Pfizer) impregnated with $5 \mu \mathrm{l}$ of $4 \%$ (w/v in $0.9 \%$ saline) Fluorogold (FG) (Fluorochrome) placed into the injury site (FG animals: T9 DF 1 week, $n=16$; 4 weeks, $n=7$; C5 Hx 1 week, $n=8 ; 3$ weeks, $n=$ 1; no FG animals: C5 Hx 3 weeks, $n=5$ ).

T9 contusions. To assess CST axon degeneration in a more severe, and clinically relevant injury paradigm (Bresnahan et al., 1991), we took advantage of a colony of rats that had received contusion injuries $\sim 1$ year previously. These animals were part of a colony of spinal cord-injured rats that had been maintained specifically to allow experiments on rats with chronic spinal cord injuries. The adult female rats $(225-250 \mathrm{~g}$ at time of surgery) received moderate contusive injuries at $\mathrm{T} 9$, using the Infinite Horizon (IH) Impactor equipped with a 2.5-mm-diameter impactor probe (Precision Systems and Instrumentation). The vertebral column was stabilized by clamping the vertebrae immediately rostral and caudal to the exposed spinal cord with stabilizing forceps. The force of the impact to the spinal cord was set at either 200 or $250 \mathrm{kdyn}$. The completeness of the injury was verified using an operating microscope; the muscles were sutured in layers, and the skin closed with wound clips. Animals were assessed for locomotor recovery after injury using the Basso, Beattie, and Breshnahan (BBB) scale for locomotor recovery (Basso et al., 1995), a 21-point scale that rates locomotor function, with 0 being complete paralysis, and 21 , normal locomotion. Nine rats were selected from the chronically injured colony that received moderate contusions (200-250 kDa) and exhibited BBB scores ranging from 10 to 11 at 1 year. This severity of injury ranges from occasional weight supported plantar steps without coordination of the forelimbs and hindlimbs (FLsHLs), to frequent weight supported plantar steps without coordination of the FLs-HLs (Basso et al., 1995). Another group of young female rats (3 months of age; $n=5$ ) received moderate contusion injuries and were killed 3 weeks after injury.

Axon degeneration controls. To assess the nature and extent of axonal degeneration in the medullary pyramid after destruction of cortical motoneurons, tissue from animals from a previous study (Strong et al., 2009) was used. Briefly, the right sensorimotor cortex was removed by aspiration in adult female rats (225-250 g). After injury, the scalp was sutured. This injury impairs motor function of the contralateral forepaw, described previously (Strong et al., 2009). Animals were allowed to survive for $4(n=6)$ or 11 weeks $(n=5)$ after injury. CST axons project exclusively within the ipsilateral medullary pyramid, so the contralateral pyramid from the 4 week time point that contains axons from the uninjured side of the cortex served as an intraanimal control for axon counts $(n=6)$.

CST tract tracing. To determine the distribution of CST axons in the medullary pyramid to accurately define the area of the pyramidal tract for axon counts, adult female rats (225-250 g at time of surgery) received unilateral cortical injections of the anterograde tracer biotin-conjugated dextran amine (BDA), 10,000 molecular weight (mini-ruby-BDA; Invitrogen) using a $10 \mu \mathrm{l}$ Hamilton microsyringe fitted with a pulled glass micropipette. BDA was injected into six sites in the sensorimotor cortex of one hemisphere at coordinates of 2.5 and $1.5 \mathrm{~mm}$ lateral from the midline, either at bregma or $1 \mathrm{~mm}$ rostral and caudal. The detailed procedure has been described previously (Anderson et al., 2005; Strong et al., 2009). Because BDA is conjugated to mini-ruby, the tracer can be visualized by fluorescence microscopy in sections without any additional staining (see Fig. $1 \mathrm{~A}$ ). Rats were killed humanely either $5 \mathrm{~d}$ after injections $(n=2)$ for assessment of labeled myelinated and unmyelinated axons in the pyramid, or $21 \mathrm{~d}$ after injection $(n=5)$. These cases were also used to assess Wallerian degeneration in the medullary pyramid after partial injury to the cortex caused by multiple penetrations of the injection micropipette. A separate set of animals from a previous study (Strong et al., 2009) that had received unilateral cortical ablation injuries also received BDA injections into the contralateral cortex $21 \mathrm{~d}$ before the end of the experiment $(n=5)$. These animals were used to quantify axon degeneration associated with minimal cortical damage from these injections.

Uninjured controls. Adult female rats were used as uninjured, unoperated controls for comparison of axon counts. Animals were killed humanely as age-matched controls both for acute $(8-12$ weeks; $n=5)$ and chronic ( 1 year; $n=5$ ) time points.

\section{Perfusions}

At the end of each experiment, animals received an intraperitoneal injection of Euthasol $(195 \mathrm{mg} / \mathrm{ml}$ pentobarbital sodium and $25 \mathrm{mg} / \mathrm{ml}$ phenytoin sodium; Delmarva Laboratories) and were transcardially perfused with either $4 \%$ paraformaldehyde in $0.1 \mathrm{M} \mathrm{PBS,} \mathrm{pH} 7.4$, for light microscope analysis, or $2 \%$ glutaraldehyde $/ 2 \%$ paraformaldehyde ( $0.1 \mathrm{M} \mathrm{caco-}$ dylate buffer, $4 \mathrm{~mm}$ magnesium sulfate, $2 \mathrm{~mm}$ calcium chloride) for electron microscope (EM) analysis.

\section{Histology}

Brains and spinal cords were dissected and postfixed in $4 \%$ paraformaldehyde in $0.1 \mathrm{~m}$ PBS, pH 7.4. Brainstems were prepared for embedding in plastic as described below. Forebrains and spinal cords were cryoprotected in 30 and $27 \%$ sucrose, respectively, frozen in TissueTek OCT (VWR International), and stored at $-80^{\circ} \mathrm{C}$ until they were sectioned. Pyramids from animals that received BDA and were perfused for EM analysis were sectioned coronally into $100 \mu \mathrm{m}$ vibratome sections and stained for BDA labeling using an avidin-biotin system combined with a peroxidase/DAB (diaminobenzidine)-nickel reaction (Vector Laboratories) before resin embedding.

Resin embedding. The brainstem was blocked by hand into $1 \mathrm{~mm}$ coronal blocks, and the block containing the main part of the pyramid (see Fig. $1 C, D$, site 3 ) was embedded in resin. Tissue was rinsed in $0.1 \mathrm{M}$ 
cacodylate buffer (Electron Microscopy Sciences) overnight and again for $15 \mathrm{~min}$ the next day. The blocks were postfixed with $1 \%$ osmium tetroxide (Electron Microscopy Sciences) in $0.1 \mathrm{M}$ cacodylate buffer for $1 \mathrm{~h}$, rinsed in double distilled $\mathrm{H}_{2} \mathrm{O}\left(\mathrm{ddH}_{2} \mathrm{O}\right)$ and en bloc stained with $0.1 \%$ uranyl acetate (Electron Microscopy Sciences) in $\mathrm{ddH}_{2} \mathrm{O}$. Blocks were then rinsed in $\mathrm{ddH}_{2} \mathrm{O}$ two times for 10 min each time, dehydrated in increasing concentrations of ethanol $(70,85,95,100,100 \%)$ for $10 \mathrm{~min}$ each, and then in propylene oxide (intermediate solvent; Electron Microscopy Sciences) two times for $10 \mathrm{~min}$ each time. Tissue blocks were then incubated in propylene oxide/Spurr's resin (1:1 mix) for $1 \mathrm{~h}$, and then in Spurr's resin (Electron Microscopy Sciences) overnight. The blocks were put in a fresh change of resin in BEEM capsules (Electron Microscopy Sciences) the next day and polymerized overnight at $60^{\circ} \mathrm{C}$.

Sectioning. Frozen brains and spinal cords were sectioned into $20 \mu \mathrm{m}$ sections on a MICROM HM505 NP Series cryostat set at $-20^{\circ} \mathrm{C}$. Brains were sectioned coronally, maintaining serial order, and sections were stored in PBS with $0.02 \%$ sodium azide in sterile 48 -well plates at $4^{\circ} \mathrm{C}$ until processing. Spinal cords were sectioned into $20 \mu \mathrm{m}$ sagittal and horizontal sections for T9 dorsal funiculus lesions and C5 hemisections, respectively, and directly mounted onto Fisherbrand Superfrost Plus glass slides (Thermo Fisher Scientific) and allowed to dry overnight at room temperature, and either stained immediately, or stored at $-80^{\circ} \mathrm{C}$ until stained for lesion verification. Medullary pyramids embedded in resin were sectioned into $1 \mu \mathrm{m}$ coronal sections using a Leica Ultracut UCT ultramicrotome (Leica Microsystems) and stained with toluidine blue O (Sigma-Aldrich; catalog \#198161) (1\% toluidine blue and 2\% sodium borate in $\mathrm{dd}_{2} \mathrm{O}$ ) at $60^{\circ} \mathrm{C}$ for $3 \mathrm{~min}$, and mounted onto Fisherbrand Superfrost Plus slides and coverslipped.

Samples from rats with unilateral cortical lesions, as well as uninjured controls, were also prepared for electron microscopy. Pyramids were embedded in resin, and ultrathin sections of $60 \mathrm{~nm}$ thickness were cut using a Leica Ultracut UCT ultramicrotome, mounted on copper grids, stained with uranyl acetate and lead citrate, and viewed on a JEOL 1400 transmission electron microscope. Images were captured using a Gatan Digital camera.

Lesion verification. Sections through lesion sites were mounted in serial order, and one section every $100 \mu \mathrm{m}$ was stained for hemotoxylin and eosin (H\&E) for assessing lesion size. Slides were washed in PBS three times for 5 min each time, and then dehydrated in increasing concentrations of ethanol for $2 \mathrm{~min}$ each $(70,95,100,100 \%)$, defatted in xylene for $5 \mathrm{~min}$, and rehydrated in decreasing concentrations of ethanol for $2 \mathrm{~min}$ each $(100,100,95,70 \%)$. Slides were then rinsed in tap water twice, stained with hemotoxylin for $5 \mathrm{~min}$, rinsed in tap water, dipped in acid alcohol, rinsed in tap water, dipped several times in ammonia water, and rinsed in tap water. Slides were then rinsed in $95 \%$ ethanol for $2 \mathrm{~min}$, and stained with eosin for $15 \mathrm{~s}$, dehydrated in 95 and $100 \%$ ethanol for $2 \mathrm{~min}$ each, defatted in xylenes three times for $3 \mathrm{~min}$ each, and then coverslipped with DPX mounting media (Sigma-Aldrich) and viewed under a bright-field microscope.

\section{Microscopy and axon quantification}

Images were captured on an Olympus AX-80 microscope (Olympus) using MagnaFire SP2.1B software (Optronics Software). To define the location of the CST axons in the pyramid, BDA-mini ruby was visualized using the fluorescence of the conjugated mini-ruby (see Fig. $1 \mathrm{~A}$ ).

Total myelinated axon counts. Toluidine blue-stained plastic sections were used to count the total number of myelinated axons in the medullary pyramid (see Fig. $1 B$ ). This was estimated by systematic random sampling (SRS) and the fractionator counting technique using the Stereo Investigator system (MicroBrightField; version 7.003 software; MBF Bioscience). Axon counts were performed blind with respect to injury paradigms and controls. The cross-sectional area of the medullary pyramid was determined by tracing its outlines at $20 \times$ magnification. Myelinated axons were then counted at high magnification $(100 \times$ under oil immersion) in $5 \times 5 \mu \mathrm{m}$ fractionator grids $\left(25 \mu \mathrm{m}^{2}\right)$ at a total of 25 sampling sites throughout the pyramid on each side. Because CST axons project longitudinally through the pyramid, the same sampling strategy can be used as for a nerve bundle; any single cross section will contain all the axons that project longitudinally through the medullary pyramid (see
Fig. $1 C, D)$. The following equation was used to estimate the total number of myelinated axons per side in the pyramid: Total axons $=[(\mathrm{NS} \div$ AS) $\times$ AP], where NS is the average number of axons per sampling site, AS is the area of each sampling site $\left(25 \mu \mathrm{m}^{2}\right)$, and AP is the area of the pyramid.

Degenerating axon counts. Toluidine blue-stained plastic sections were used to count degenerating axons; counts were performed blind with respect to injury paradigms and controls. The entire cross section of the medullary pyramid was scanned at high magnification $(120 \times$, under oil immersion) to count the total number of axons displaying degenerative changes. The main criterion for classifying an axon as degenerating was a dark dense cytoplasm (Griffin et al., 1995; Rosenbluth, 1995); supplemental indicators included myelin breakdown and demyelination (Rosenbluth, 1995). For cases with cortical lesions where the number of axons displaying degenerative changes was too high to count using this method, the SRS method with fractionator counting (described above) was used, with uninjured controls counted in the same fashion to correct for between group comparisons using the two different counting methods (direct counting vs SRS counting). It is important to note here that direct counting through the microscope allowed focusing up and down, which allowed a better assessment of the morphology of the axons. In contrast, with the SRS method, counts are made from the computer screen, which decreases the resolution of the image.

Assessment of axonal atrophy. To assess possible axon atrophy, we measured the cross-sectional area of axons in the pyramid using the same toluidine blue-stained plastic sections described above, a technique that has been shown previously to be a measure of axonal atrophy after injury (Gold et al., 1992). High-magnification images (120×, under oil immersion) of pyramids were obtained and assessed using NIH ImageJ (image processing and analysis). Representative samples from C5 hemisection (3 weeks; $n=2$ ), T9 contusions ( 3 weeks, $n=1 ; 1$ year, $n=2$ ), and uninjured controls $(8-12$ weeks, $n=2 ; 1$ year, $n=1)$ were assessed for axonal atrophy. A total of three images per side (lateral, middle, medial) was taken for each pyramid (see Fig. $8 A$ ), with three sampling sites assessed per image, for a total of nine sites. Axons at each site were assessed in $5 \times 5 \mu \mathrm{m}$ fractionator grids $\left(25 \mu \mathrm{m}^{2}\right)$, and only axons within the accepted boundaries of the grid were analyzed for area. Using ImageJ, the perimeters of axons within the sampling area were traced, including the myelin sheath.

Quantification of BDA-labeled axons. Electron microscope images of axons in the pyramid of animals $5 \mathrm{~d}$ after cortical BDA injections $(n=2)$ were quantified to determine whether unmyelinated axons originate in the sensorimotor cortex. Images were taken from the center of the pyramid and quantified based on electron density indicative of positive BDA labeling. BDA-labeled myelinated and unmyelinated axons were counted in each sample site.

\section{Statistical analysis}

Data were analyzed for statistical significance between time points, injury type, and controls using a one-way ANOVA with Bonferroni's multiplecomparison test for post hoc analysis using Prism 4 for Macintosh. Graphs are plotted as the average from each group tested, with the error bars representing the SD.

\section{Results \\ Quantitative assessment of myelinated axons in the medullary pyramid}

CST axons that project from the sensorimotor cortex to the spinal cord pass through the ventral medulla in the pyramid (Kuypers, 1981). Thus, for purposes of axon counting, the pyramid can be considered as a nerve bundle with defined boundaries. The ventral and lateral boundaries of the medullary pyramid are obvious, but the dorsal boundary with the ventral medulla is less so. To define the boundaries of the CST in the medullary pyramid, CST axons were labeled by injecting mini-ruby-BDA into the sensorimotor cortex of one hemisphere (Fig. $1 A$ ). The region occupied by BDA-labeled axons was used as a guide to define the region 

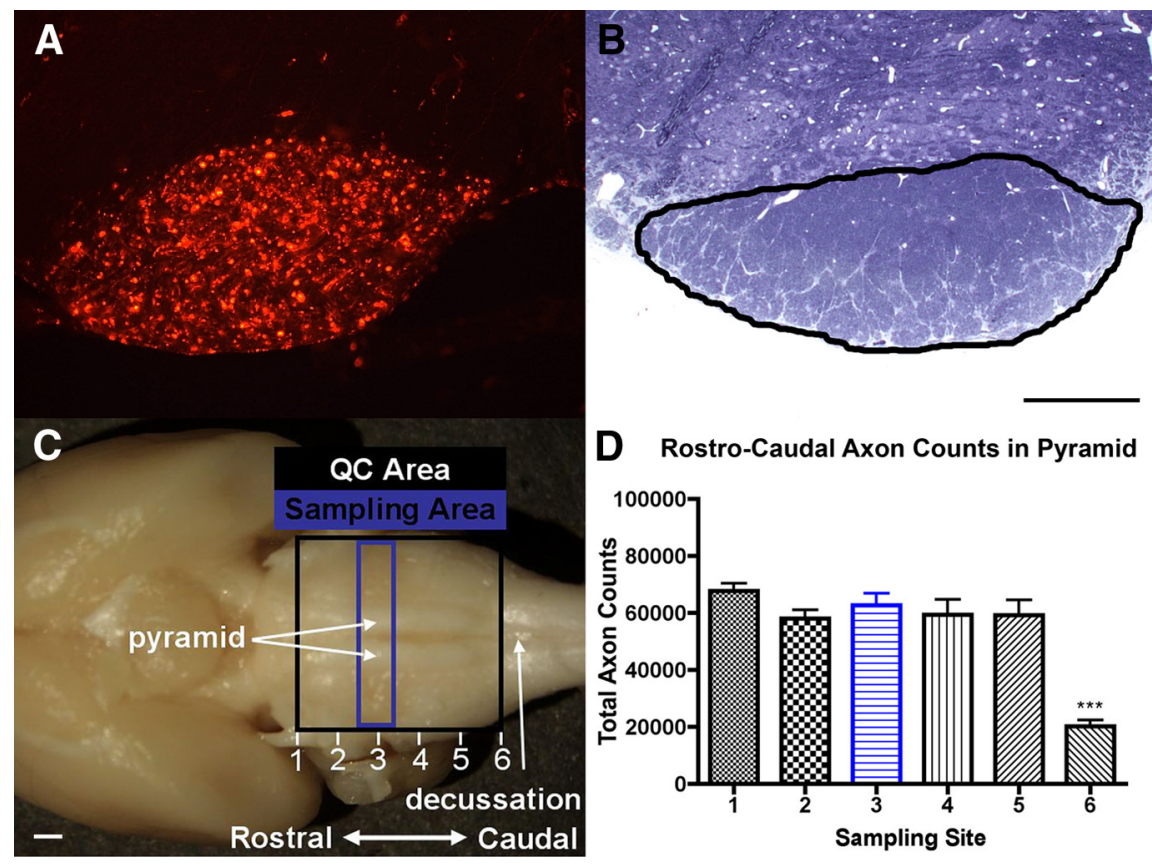

Figure 1. Development of axon counting method. $\boldsymbol{A}$, Anterograde tract tracing with BDA-mini-ruby to outline the border of CST axons in the pyramid. $\boldsymbol{B}$, Toluidine blue-stained plastic sections of the pyramid with the region of CST axons outlined to define the area of the pyramid for axon counts. $C$, Ventral view of rat brain to illustrate the sampling strategy of counting axons in the pyramid. A total of six sampling sites were assessed in $1200 \mu \mathrm{m}$ intervals for quality control counts of total axons from the rostral-most region where axons enter the pyramid (site 1) to the caudal-most region where axons begin to decussate into the spinal cord (site 6). D, Quantification of axon counts, with total axons per section on the $y$-axis shows no significant difference between sites 1 and 5 ; however, there is a significant decrease in total axons at site $6(p<0.001)$, where the axons begin to decussate. All axon counts for the remainder of the study were taken from site 3 (sampling area). Scale bar: $\boldsymbol{A}, \boldsymbol{B}, 200 \mu \mathrm{m} ; \boldsymbol{C}, 1 \mathrm{~mm}$. Data are mean \pm SD. *** $p<0.001$.

of interest in the toluidine blue-stained plastic cross sections for axon counts (Fig. $1 B$ ).

One issue for the quantitative analysis was to determine whether small differences in the rostrocaudal location of the sample cross section through the medullary pyramid would affect axon counts. To assess this, sample sections were taken at 1200 $\mu \mathrm{m}$ intervals starting from the caudal portion of the pons to the border between the medulla and the spinal cord where the decussation occurs (a total of six sampling sites) (Fig. 1C). Counts of myelinated axons were performed at each site (Fig. 1D). There were no significant differences in myelinated axon number between any of the five rostral sampling sites, although axon number was slightly higher in the rostral-most site. This may be explained by corticonuclear fibers leaving the pyramid as this does correspond to the region where the facial nucleus and nucleus ambiguous are located near the pons-medulla border (Zimmerman et al., 1964; Reh and Kalil, 1981; Wiesendanger, 1981; FitzGerald et al., 2007). Fewer axons were present at the caudal site (site 6), which is just rostral to the pyramidal decussation $(p<0.001)$; this is probably a reflection of the fact that CST axons are beginning to leave the pyramid to ascend dorsally and cross over before joining the dorsal CST in the spinal cord. Given the consistency of axon counts in different rostrocaudal locations, subsequent analyses were performed by taking the $1 \mathrm{~mm}$ block from approximately site 3 .

\section{Validation of approach: CST axon degeneration in the medullary pyramid after cortical lesions}

Death of cortical neurons after direct trauma will cause Wallerian degeneration of CST axons throughout their course. To validate the use of the medullary pyramid as a sampling site, medullary pyramids were assessed for degenerating and intact axons after varying degrees of direct cortical injury (complete ablation and more limited damage caused by six injections of BDA for tract tracing).

At 4 weeks after cortical ablations that extended from $0.5-4 \mathrm{~mm}$ lateral to the midline and 4 and $4.5 \mathrm{~mm}$ rostral and caudal to bregma (Fig. 2C), large numbers of axons in the medullary pyramid exhibited classical signs of Wallerian degeneration characterized by darkly stained axoplasm, breakdown of myelin, and subsequent demyelination (Figs. $3 B, D, 4 B$ ). Wallerian degeneration was still prominent 11 weeks after cortical ablation (Fig. 5B). Previous studies (Strong et al., 2009) have shown that these cortical lesions successfully ablated the region in the sensorimotor cortex that contains the cells of origin of CST that project to lumbar segments as verified by the elimination of neurons labeled by Fluorogold after injections into the lumbar spinal cord. Degenerating axons were also evident in the medullary pyramid in animals that received injections of BDA-mini-ruby into the sensorimotor cortex (data not shown) (see counts below), although fewer were degenerating than after cortical ablations. This suggests that this method of assessing degeneration is sensitive to even a small amount of damage to the cortex.

Assessment of the area of the pyramid after cortical injuries demonstrates that, 11 weeks after cortical ablation, there is a significant decrease in the area of the pyramid $(p<0.01)$ by $\sim 30 \%$ from the uninjured controls (Fig. $3 E$ ). This confirms previous studies in which cortical ablation lesions led to a time-dependent decrease in pyramid area, and our time point of 11 weeks with $30 \%$ shrinkage confirms what has been shown at that time point (Dunkerley and Duncan, 1969). However, this only explains a decrease in the area of the pyramid and cannot definitively conclude whether this shrinkage is caused by a loss of axons or a breakdown of the structure of the pyramid.

SRS-based counts of the axons (Fig. $3 F$ ) revealed no significant difference in total axon numbers (intact and degenerating) between uninjured controls $(72,380.25 \pm 9430.44)$ when compared with the cortical injections $(67,277.01 \pm 8956.30 ; 92.95 \pm 12.37 \%$ of total $)$ or cortical ablation at 4 weeks $(60,334.72 \pm 9558.45 ; 83.36 \pm 13.21 \%$ of total) after injury. Thus, degenerating axons are not rapidly removed. There were significantly fewer total axons in the medullary pyramids at 11 weeks $(51,682.81 \pm 12,486.26 ; 71.40 \pm$ $17.25 \%$ of total; $p<0.05$ ), and this decrease appears to correlate with the shrinkage of the pyramid. As expected, there were fewer non-degenerating axons after cortical ablation compared with uninjured controls (uninjured, 68,891.30 \pm 9977.36, $95.18 \pm$ $13.78 \%$ of total; 4 weeks after ablation, 38,265.82 \pm 7725.77 , $52.87 \pm 10.67 \%$ of total, $p<0.001 ; 11$ weeks after ablation, $34,950.83 \pm 8904.11,48.29 \pm 12.30 \%$ of total, $p<0.001)$. There were also fewer non-degenerating axons in rats with cortical in- 
A Cx Schematic

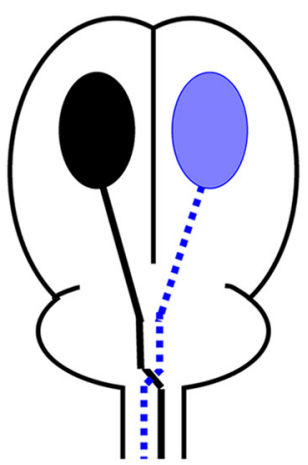

D T9 DF Schematic

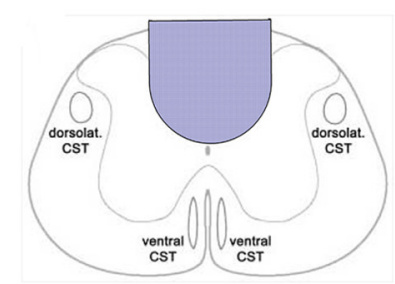

G C5 Hx Schematic

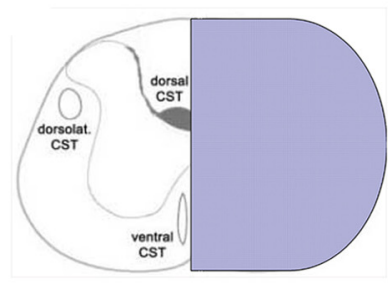

B Cx Dorsal View

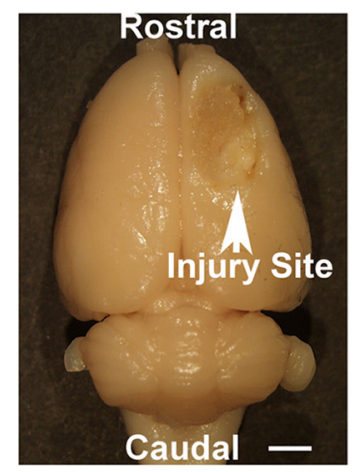

E T9 DF Dorsal View Injury Site

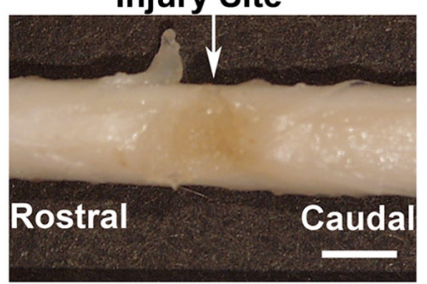

H C5 Hx Dorsal View

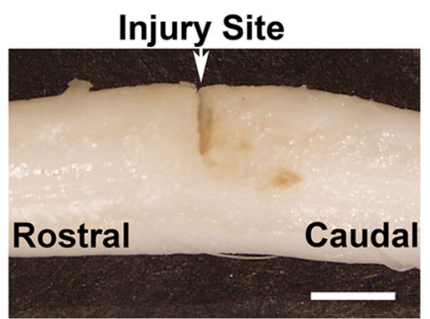

C Cx Coronal Section - H\&E

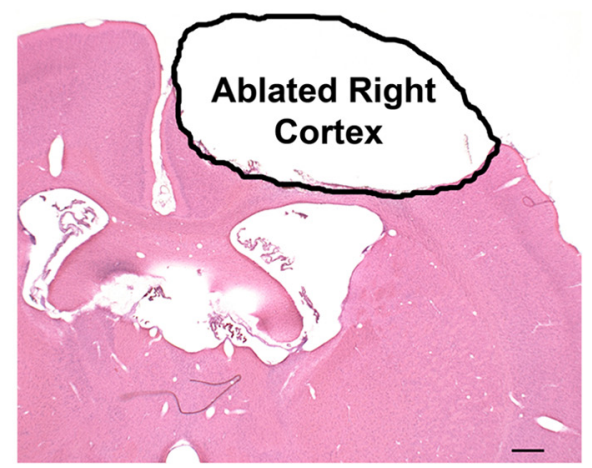

F T9 DF Sagittal Section - H\&E

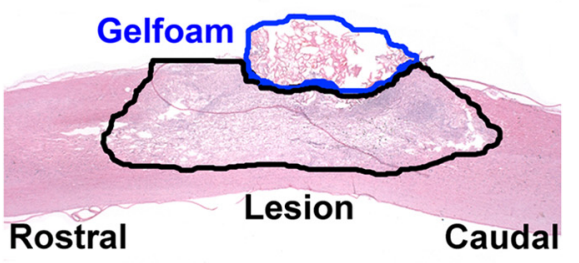

I C5 Hx Horizontal Section - H\&E

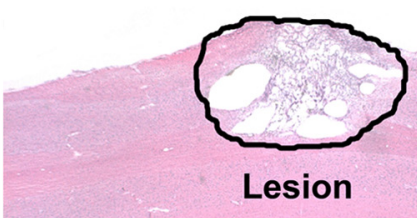

Rostral

\section{Caudal}

Figure 2. Lesion verification. $\boldsymbol{A}-\boldsymbol{I}$, Representative images for lesions from cortical ablations (Cx) $(\boldsymbol{A}-\boldsymbol{C})$, T9DF lesions $(\boldsymbol{D}-\boldsymbol{F})$, and $\mathbf{C} 5 \mathrm{Hx}(\boldsymbol{G}-\boldsymbol{I})$. $\boldsymbol{A}, \boldsymbol{D}, \mathbf{G}, \mathbf{S}$ chematic diagrams for each injury paradigm to illustrate the extent of CST that is to be targeted for each injury, where $\boldsymbol{A}$ illustrates ablation of the right sensorimotor cortex to remove the CST cell bodies as a positive degeneration control. $\boldsymbol{D}$ illustrates bilateral transection of the dorsal CST at T9, and $\boldsymbol{G}$ illustrates unilateral transection of the crossed dorsal, dorsolateral, and uncrossed ventral CST. $\boldsymbol{B}, \boldsymbol{E}, \boldsymbol{H}$, Representative dorsal view images of the lesions after perfusion and dissection to confirm location of each lesion. $\boldsymbol{C}, \boldsymbol{F}, \boldsymbol{I}$, Representative $20 \mu \mathrm{m}$ sections of lesion sites after hematoxylin and eosin (H\&E) for lesion verification. $\boldsymbol{C}$, $\boldsymbol{C X}$ lesions assessed in coronal sections successfully ablated the majority of the sensorimotor cortex in all animals tested. $\boldsymbol{F}$, T9 DF in sagittal sections demonstrated that this injury consistently transected the entire dorsal column, extending laterally between the dorsal horn on each side, and ventrally past the central canal for complete bilateral transection of the dorsal CST. Gelfoam was present in some of the lesion sites because of retrograde tracing at the time of injury. $I$, C5 Hx horizontal sections confirmed these lesions were all complete in the dorsal CST on the right side of the spinal cord, and the majority of them were also complete in the dorsolateral CST and uncrossed ventral CST, with some lesions affecting the contralateral side of the spinal cord. Scale bars: $\boldsymbol{B}, \boldsymbol{E}, \boldsymbol{H}, 2$ mm; $\boldsymbol{C}, \boldsymbol{F}$, $I, 500 \mu \mathrm{m}$.

jections $(55,932.38 \pm 12,326.47 ; 77.28 \pm 17.03 \%$ of total in control rats), but this difference was not statistically significant.

It is noteworthy that complete ablation of the sensorimotor cortex led to the loss of only $\sim 50 \%$ of the axons in the medullary pyramid by 11 weeks, suggesting that $\sim 50 \%$ of the myelinated axons in the pyramid come from areas other than the sensorimotor cortex. This is consistent with previous studies (Miller, 1987) (see Discussion).

Counts revealed abundant degenerating axons 4 and 11 weeks after ablation $(11,456.77 \pm 3368.15,15.83 \pm 4.65 \%$ of total; $10,838.02 \pm 1661.20,14.97 \pm 2.30 \%$ of total); however, these two time points were not significantly different from each other. The number of degenerating axons after cortical ablation was significantly higher than what was seen after BDA injections $(p<0.01$ and $p<0.001$, respectively; $4085.13 \pm 3144.52 ; 5.64 \pm 4.34 \%$ of total).

SRS-based assessments of the medullary pyramids from uninjured controls did reveal a small and variable number of possibly degenerating axons $(550.77 \pm 507.04 ; 0.76 \pm 0.70 \%$ of total $)$. However, counts of the same samples using direct microscopic evaluation yielded values for degenerating axons that were approximately two orders of magnitude lower; we feel the lower counts are more accurate given the increased resolution of morphology when the tissue is examined at high power through the microscope. Importantly, even the higher number of possibly degenerating axons from SRS-based analyses represents only a fraction of a percentage of the total. A caveat is that there may also be false positives in the counts of from animals with cortical lesions that would not be scored as degenerating if the counts were done by direct microscopic visualization. However, the degree of degeneration that is seen with SRS-based counting is proportional to the amount of damage in the cortex.

Thus, even with the differences between SRS-based and direct counts, it is clear that degenerating axons are easy to detect in the pyramid when present after a cortical lesion, validating the 


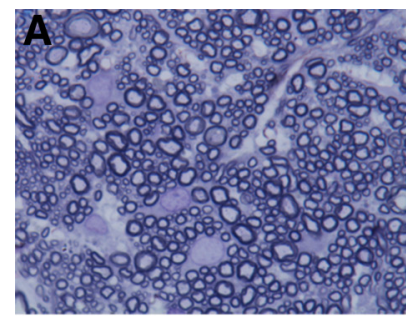

Healthy Axons

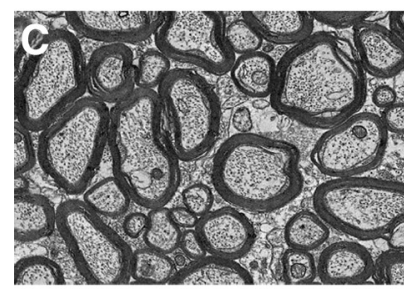

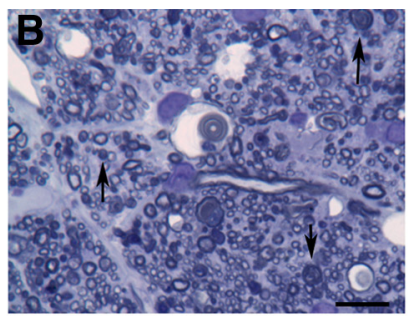

Degenerating Axons

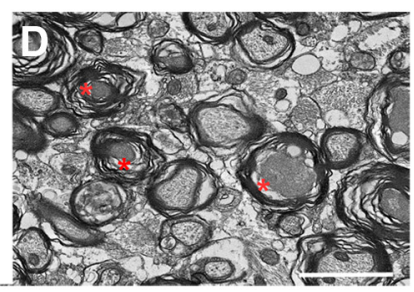

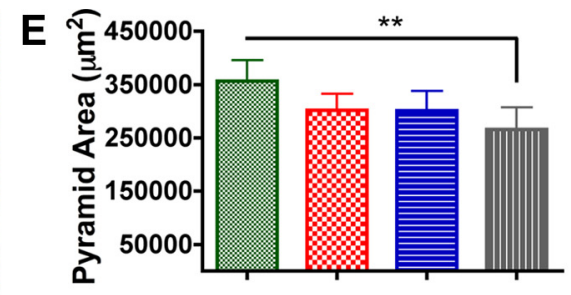

$F$

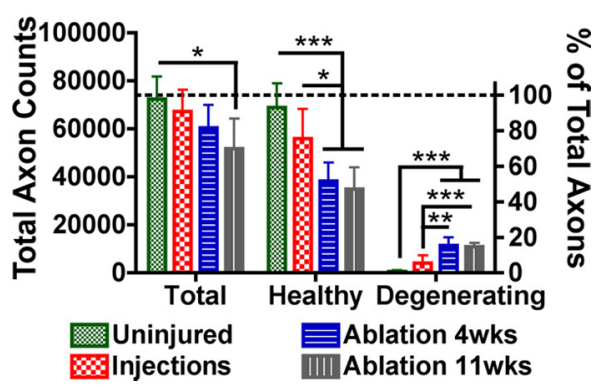

Figure 3. Identifying degenerating axons. $\boldsymbol{A}, \boldsymbol{B}$, Light micrographs $(100 \times)$ of toluidine blue-stained plastic sections from pyramids in healthy, uninjured animals $(\boldsymbol{A})$ compared with degenerating axons seen after a cortical ablation (Cx) lesion $(\boldsymbol{B})$. C, D, Electron micrographs $(2500 \times)$ of pyramid axons in uninjured $(\boldsymbol{C})$ compared with cortical ablation $(\boldsymbol{D})$. Healthy axons display clear axoplasm with a continuous myelin ring $(\boldsymbol{A}, \boldsymbol{C})$, which is confirmed at higher magnification to demonstrate the integrity of the neurofilaments and microtubules in the axoplasm $(\boldsymbol{D})$. Axons that display degenerative changes show darkly stained axoplasm and breakdown of the myelin sheath ( $\boldsymbol{B}$, black arrows), which is also confirmed at high magnification showing degeneration of the axoplasm components and myelin breakdown ( $\boldsymbol{D}$, red asterisks). The graphs in $\boldsymbol{E}$ and $\boldsymbol{F}$ demonstrate that we can quantify a significant loss of axons in the pyramid, both by shrinkage of the whole pyramid by 11 weeks after $(x$ lesion $(p<0.01)(\boldsymbol{E})$, as well as a significant loss of total $(p<0.05)$ and healthy axons $(p<0.001)$, corresponding to a significant increase in degenerating axons $(p<0.001)$ after $C x$ lesions $(\boldsymbol{F})$. Scale bars: $\boldsymbol{A}, \boldsymbol{B}, 10 \mu \mathrm{m} ; \boldsymbol{C}, \boldsymbol{D}, 2 \mu \mathrm{m}$. Data are mean \pm SD. ${ }^{* *} p<0.001,{ }^{* *} p<0.01,{ }^{*} p<0.05$.

approach for assessing CST axon degeneration after spinal cord injury.

\section{Absence of degeneration of CST axons after bilateral dorsal funiculus lesion at $\mathrm{T} 9$}

A previous study reported the retrograde degeneration of $\sim 20 \%$ of cortical motoneurons in young male rats after a T9 DF injury (Hains et al., 2003). Cortical motoneurons exhibited signs of cell death (caspase activation) by 1 week, and there was a $20 \%$ decrease in the number of retrogradely labeled cortical motoneurons by 4 weeks. If $20 \%$ of the cortical motoneurons die, then $20 \%$ of the axons in the medullary pyramid will also die, which should be detectable as Wallerian degeneration and by decreases in the number of intact axons.

Surprisingly, however, we detected essentially no sign of degenerating axons in the medullary pyramid 1 week after DF lesions at $\mathrm{T} 9$ that ablated the dorsal column bilaterally, with the lesion extending ventrally into the gray matter past the central canal, and laterally into the dorsal horn (for an example of the lesion, see Fig. 2 F). Based on the results of Hains et al. (2003), there should be extensive retrograde degeneration, but CST axons in the medullary pyramid appeared entirely normal (Fig. 4C). Degenerating axons were also not seen at 4 weeks after injury (Fig. 4D), by which time Hains et al. (2003) report substantial loss of retrogradely labeled cortical motoneurons (Hains et al., 2003). At both 1 and 4 weeks, a very small number of axons stained darkly enough that it could not be excluded that they were degenerating, but no axons exhibited definitive signs of degeneration (dark axoplasm, separation of myelin, and myelin unraveling). Moreover, the number of axons with dark cytoplasm (possibly degenerating) was no higher than in uninjured control rats (see quantitative analysis below).

Quantitative analysis of pyramidal tract axons by high magnification microscopic scanning after the T9 DF at 1 and 4 weeks after injury confirmed the absence of degenerating axons. Only a tiny fraction of the total axons in the pyramid were scored as possibly degenerating at either 1 week $(6.7 \pm 4.2 ; 0.0108 \pm$ $0.0066 \%$ of total $)$ or 4 weeks after injury $(4.8 \pm 2.6 ; 0.0075 \pm$ $0.0040 \%$ of total) (Fig. 6C). Approximately the same number of possibly degenerating axons were seen in the pyramids from uninjured controls $(3.63 \pm 1.4 ; 0.0058 \pm 0.0021 \%$ of total) (Fig. $6 C)$. The values from injured and control rats did not differ significantly.

SRS-based counts of total axons also revealed no axon loss at either time point ( 1 week, 65,851.86 \pm 6461.02; 4 weeks, $63,912.89 \pm 7527.20$ ) (Fig. $6 E$ ) compared with uninjured controls $(62,978.35 \pm 7283.65)$ (Fig. $6 E$ ). These results exclude the possibility that axons that die as a result of retrograde degeneration of the cell body are removed quickly and thus are not counted as degenerating. This is also confirmed by the fact that the area of the pyramid does not change significantly after injury (uninjured, 313,106.33 \pm 45,693.33; 1 week, 296,602.88 \pm 33,129.22; 4 weeks, 320,737.64 \pm 50,309.90) (Fig. 6A). The lack of axonal degeneration or axon loss indicates that the cell bodies of pyramidal tract axons do not die after DF lesions at T9, at least up to 4 weeks after SCI.

\section{Absence of degeneration of CST axons after lateral hemisections at $\mathbf{C 5}$}

Given the lack of detectable Wallerian degeneration or axon loss after DF lesions at T9, it was of interest to assess the consequences of a lesion that was more complete (that is, destroyed a greater proportion of CST axons at the injury level) and more proximal to the cell bodies (which would be expected to cause a greater degree of retrograde degeneration than a more distal injury) (Liu, 1955; Beresford, 1965). Lateral hemisections are more complete than DF lesions in that they destroy the main contingent of CST axons in the dorsal funiculus as well as CST axons in the dorsolateral and ventral CST, albeit unilaterally. Additionally, a lesion at C5 is much more proximal to the cell bodies than a lesion at T9.

Lesions at $\mathrm{C} 5$ were variable in extent (Fig. $7 \mathrm{~A}, \mathrm{C}$; supplemental Fig. 1, available at www.jneurosci.org as supplemental material). 

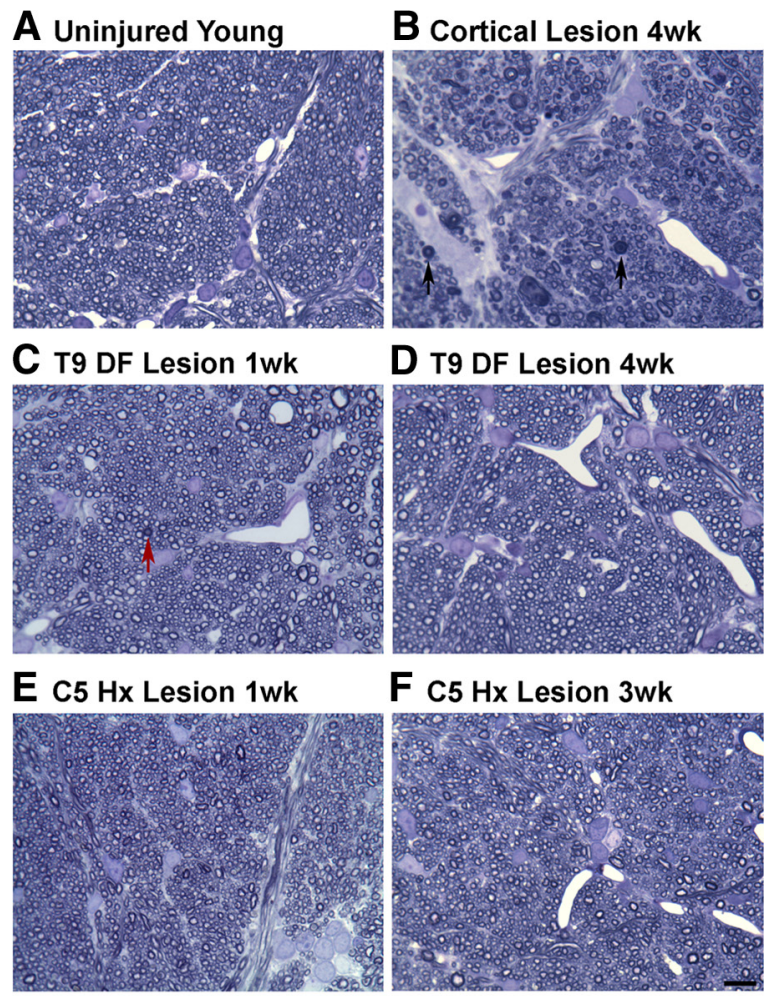

F C5 Hx Lesion 3wk
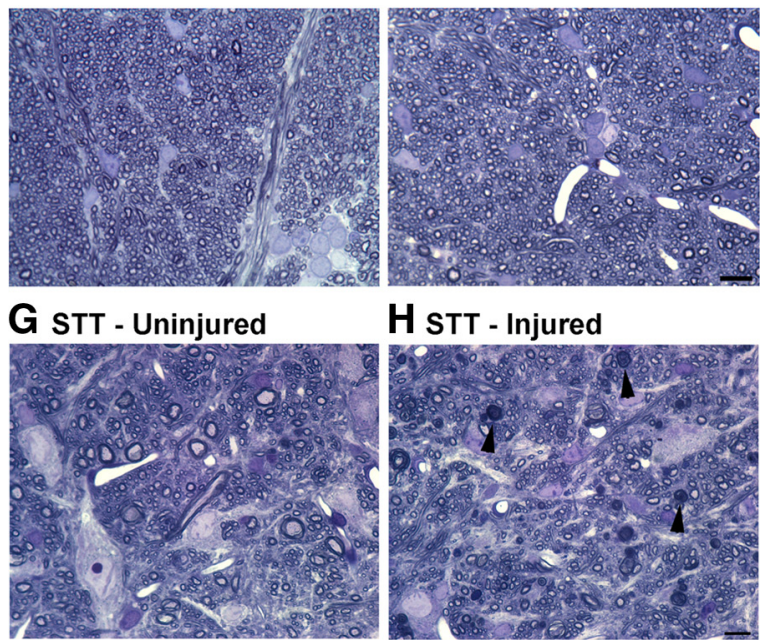

Figure 4. Histological assessment of myelinated axons in the pyramid after acute transection SCl. $\boldsymbol{A}, \boldsymbol{B}$, Representative sections of toluidine blue-stained plastic sections $(100 \times)$ to assess axon health and morphology after injury. Uninjured $(\boldsymbol{A})$, healthy axons have clear axoplasm and a continuous myelin ring, whereas degenerating axons, seen after cortical ablation lesion ( $\boldsymbol{B}$ ) causes significant amounts of detectable degeneration, have darkly stained axoplasm and myelin degradation (black arrow). $\mathbf{G}, \boldsymbol{H}$, Light micrographs $(120 \times)$ of toluidine bluestained plastic sections from the brainstem to show integrity of the ascending spinothalamic tract (STT) lateral to the pyramid in uninjured $(\boldsymbol{G})$ and degenerating $(\boldsymbol{H})$ axons after $\mathrm{Hx} \mathrm{SCl}$, which induces Wallerian degeneration (black arrowheads). The axons in the pyramid after a T9 DF lesion, 1 week $(\boldsymbol{C})$ and 4 weeks $(\boldsymbol{D})$, as well as after a $\mathbf{C} 5$ Hx injury, 1 week $(\boldsymbol{E})$ and 3 weeks $(\boldsymbol{F})$, did not result in significant degeneration such as the $C x$ lesion. There are a few axons that exhibit degenerative changes ( $\boldsymbol{C}$, red arrow) and are quantified in Figures 6 and 7 , respectively. Scale bars, $10 \mu \mathrm{m}$ (for all panels); (in $\boldsymbol{F}$ ) $\boldsymbol{A}-\boldsymbol{F}$, images taken at $100 \times$; (in $\boldsymbol{H}$ ) $\boldsymbol{G}, \boldsymbol{H}$, images taken at $120 \times$.

Lesions were reconstructed by assessing horizontal sections through the lesion site at $100 \mu \mathrm{m}$ intervals and representing these on schematic cross sections. In the reconstructed images, lesions in different animals are shown in gray superimposing images from different animals. In this way, the darkest shade of gray indicates the areas damaged in all animals; the lightest shade of gray illustrates the area damaged in one animal only (supplemental Fig. 1, gray area, available at www.jneurosci.org as supplemental material). The quantitative analyses were limited to cases in which lesions extended to the midline so as to completely destroy the ipsilateral dorsal CST (Fig. 2I) ( 1 week, $n=8$; 3 weeks, $n=6$ ) and also included cases in which lesions extended over to the contralateral side. For axon counts after C5 hemisections, data

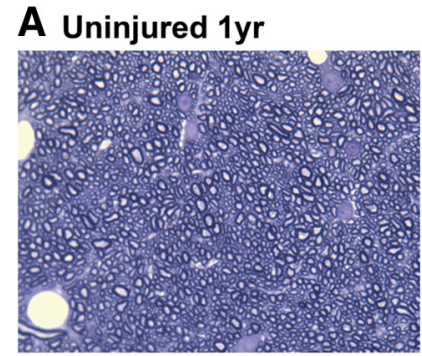

C T9 contusion $3 w k$

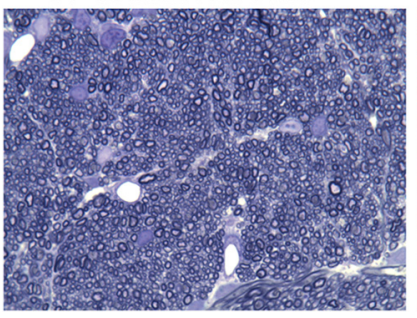

\section{B Cortical lesion 11wk}

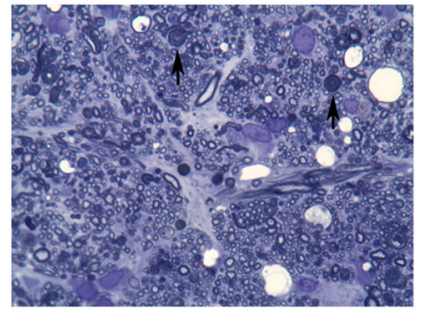

D T9 contusion $1 \mathrm{yr}$

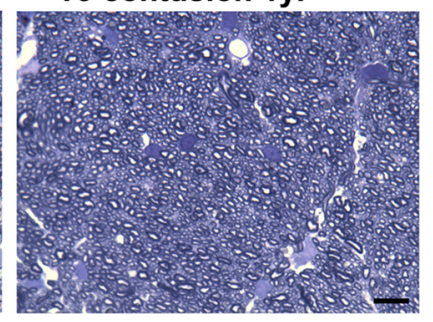

Figure 5. Histological assessment of myelinated axons in the pyramid after contusive and chronic SCl. Representative sections of toluidine blue-stained plastic sections to assess axon health and morphology after a more severe type of injury at chronic time points. Uninjured animals aged 1 year $(\boldsymbol{A})$ have healthy axons with clear axoplasm and a continuous myelin ring, compared with cortical lesion positive controls displaying widespread axon degeneration ( $\boldsymbol{B}$, black arrows). The axons in the pyramid after a T9 contusion both at 3 weeks $(\boldsymbol{C})$ and even up to 1 year $(\boldsymbol{D})$ after injury do not show any detectable signs of degeneration to suggest their cell bodies had died as a result of the injury. Scale bar, $10 \mu \mathrm{m}$.

are organized into three categories: lesioned side refers to the pyramids contralateral to the hemisection containing damaged axons from the crossed dorsal and dorsolateral CST; unlesioned side refers to the pyramids ipsilateral to the hemisection that include mainly undamaged axons, but also includes axons of the uncrossed ventral CST and axons affected by over-hemisections; uninjured refers to control animals with no lesions.

Even though lesions were predominantly unilateral, the pyramid on the side contralateral to the lesion that would contain the majority of the injured axons (lesioned side) was indistinguishable from the side where there are mostly uninjured axons that served as an internal control (unlesioned), both qualitatively and quantitatively. Importantly, however, the sides were distinguishable based on the presence of degenerating axons lateral to the pyramid indicating Wallerian degeneration of spinothalamic and spinoreticular axons (Fig. $4 \mathrm{H}$ ); degenerating axons were present only ipsilateral to the lesion (compare with Fig. 4G). This internal control again confirms that Wallerian degeneration can be detected in these sections when present.

Histologically, CST axons in the medullary pyramid appeared entirely normal, at both 1 week (Fig. $4 E$ ) and 3 weeks after C5 hemisections (Fig. $4 F$ ). This was true whether the $\mathrm{C} 5$ hemisection injury was limited to one side, or extended over the midline to affect the contralateral spinal cord (for analysis of the relationship between lesion size and axon counts, see supplemental Fig. 2, available at www.jneurosci.org as supplemental material). There was no significant difference in the area of the pyramid at 1 week (lesioned, 316,825.40 $\pm 33,210.00 \mu \mathrm{m}^{2}$; un-

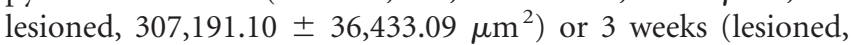
$294,301.13 \pm 37,521.80 \mu \mathrm{m}^{2}$; unlesioned, 292,127.25 \pm 41,377.14 $\mu \mathrm{m}^{2}$ ) after injury compared with uninjured controls (Fig. $7 B$ ), and counts by high magnification microscopic scanning again revealed only a fraction of a percentage of the total that exhibited possible signs of degeneration in either pyramid at 1 week (lesioned, $4.25 \pm 2.76,0.0067 \pm 0.0044 \%$ of total; unlesioned, 


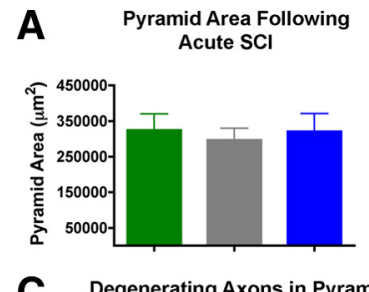

C Degenerating Axons in Pyramid Following Acute $\mathrm{SCl}$

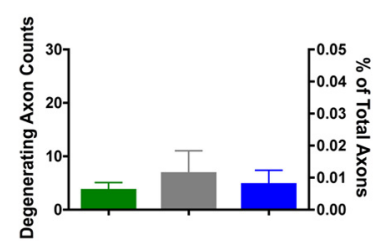

E

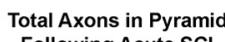
Following Acute SCl
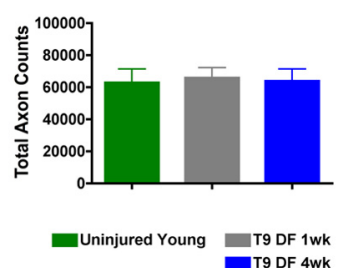
Pyramid Area Following
Chronic SCl
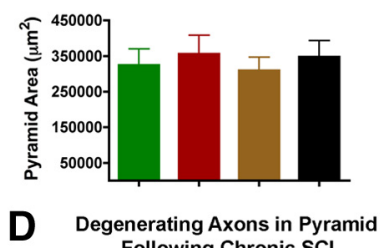
Following Chronic SCI

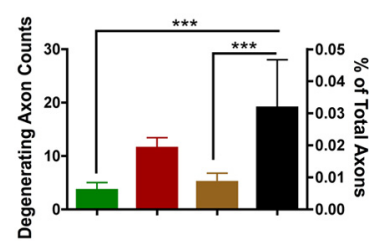

F

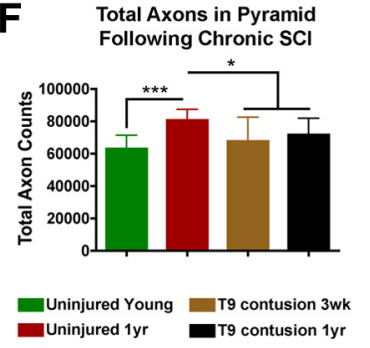

Figure 6. Quantification of axon degeneration in the pyramid after acute and chronic SCl. Quantification of the area $(\boldsymbol{A}, \boldsymbol{B})$ of the medullary pyramid, as well as degenerating $(\boldsymbol{C}, \boldsymbol{D})$ and total myelinated axons $(\boldsymbol{E}, \boldsymbol{F})$ after either acute $(\boldsymbol{A}, \boldsymbol{C}, \boldsymbol{E})$ or chronic $(\boldsymbol{B}, \boldsymbol{D}, \boldsymbol{F}) \mathrm{SCl}$, compared with age-matched controls. Neither level nor time after injury has a significant affect on axons in the pyramid at the acute time points between 1 and 4 weeks after T9 DF injuries in the spinal cord. This is demonstrated by the fact that there is no shrinkage of the pyramid after injury $(\boldsymbol{A})$ or any quantifiable signs of degenerating axons $(\boldsymbol{C})$ or loss of axons $(\boldsymbol{E})$ at these shorter survival time periods. Additionally, contusive $\mathrm{SCl}$ does not cause a significant amount of pyramid shrinkage, even up to 1 year after injury $(\boldsymbol{B})$. There are significantly more degenerating axons 1 year after injury $(p<0.001)$, but this is only a fraction of a percentage of the total axons in the pyramid $(\boldsymbol{D})$, and may be explained by the fact that there are significantly more total axons in the uninjured aged animals $(p<0.001)$ compared with their younger counterparts $(\boldsymbol{F})$. There is no significant difference between the young uninjured control and either the 3 week or 1 year time points after $\mathrm{SCl}$; however, there is a significant decrease in total myelinated axons at 1 year compared with the 1-year-old control ( $p<0.05$ ), but not compared with the younger SCl time point, suggesting that axons are not lost over time as a result of injury, but that the increase in axon number is affected by $\mathrm{SCI}(\boldsymbol{F})$. Data are mean \pm SD. ${ }^{* * *} p<0.001,{ }^{*} p<0.05$.

$5.38 \pm 3.70,0.0085 \pm 0.0059 \%$ of total) (Fig. $7 D$ ) or 3 weeks (lesioned, $5.38 \pm 2.56,0.0085 \pm 0.0041 \%$ of total; unlesioned, $5.25 \pm 1.75,0.0083 \pm 0.0028 \%$ of total) (Fig. $7 D$ ) after injury.

Also consistent with the results after T9 DF lesions, SRS-based counts of total axons revealed no axon loss between sides after C5 lateral hemisections at either the 1 week (lesioned, 65,153.84 \pm 6325.85; unlesioned, $64,248.77 \pm 9677.55)$ or 3 week time points (lesioned, 59,943.57 \pm 10,033.38; unlesioned, 60,939.77 \pm 6865.39) (Fig. 7D). Thus, even when the injury to CST axons occurs more proximal to the cell bodies in the cervical region, axons in the medullary pyramid exhibit no signs of degeneration and there is no indication of axon loss, indicating that cortical motoneurons have not died at least up to 3 weeks after injury.

\section{SCI does not cause delayed degeneration of CST axons in the} pyramid but may affect age-dependent myelination

Axons must die eventually after death of the cell body, but it is possible that the degeneration of the axon as a result of retrograde cell death takes longer than the Wallerian degeneration that occurs after physical destruction of the cell body or physical separation of the distal axon from the cell body. Accordingly, we

assessed whether there was evidence of axon degeneration or axon loss in the medullary pyramid 1 year after moderate contusion injuries at T9, which completely destroy the dorsal funiculus in the same way as a DF transection.

Again, there were only a few axons in the medullary pyramid that exhibited any sign of Wallerian degeneration in the rats with chronic T9 contusion injuries (Fig. 5D) compared with their agematched controls (Fig. 5A). Interestingly, counts did reveal that, at 1 year after a T 9 contusion, the number of possibly degenerating $(19.31 \pm 9.26 ; 0.024 \pm 0.012 \%$ of total $)$ axons in the medullary pyramid is significantly greater than the number of possibly degenerating axons 3 weeks after a DF lesion in young adult rats $(5.13 \pm 1.64 ; 0.006 \pm 0.002 \%$ of total; $p<0.001)$ (Fig. $6 D)$. Uninjured aged control rats also had a greater number of axons in the medullary pyramid exhibiting signs of degeneration (11.50 \pm $1.91 ; 0.014 \pm 0.002 \%$ of total), suggesting that these signs of degeneration are attributable to aging, and not the spinal cord injury (Fig. 6D). It is important to emphasize, however, that even in aged rats, degenerating axons represent only a fraction of a percentage of the total axons in the pyramid.

Interestingly, counts revealed that the older, uninjured control rats also have significantly more total myelinated axons (Fig. $6 F)$ in the medullary pyramid $(80,374.2 \pm 6829.9)$ than the younger, uninjured controls $(62,949.7 \pm 8419.7 ; p<0.001)$. Additionally, rats that had received moderate $\mathrm{T} 9$ contusion injuries 1 year previously had significantly fewer myelinated axons $(71,638.1 \pm 10,151.0 ; p<0.05)$ after injury, compared with the older uninjured controls. However, there was no difference in total myelinated axons compared with the younger, uninjured controls, or between 3 weeks $(67,593.6 \pm 14,960.0)$ and 1 year after contusion injury (see above). This is also confirmed in the measures of total area of the pyramid, which does not change significantly after injury (uninjured, 1 year: 355,583.2 \pm 52,596.2; T9 contusion, 3 weeks: 309,511.5 \pm 37,491.0; T9 contusion, 1 year: $347,553.3 \pm 45,643.6$ ) (Fig. 6B).

Together, these data suggest that there is no loss of myelinated axons after moderate contusion injuries, but the injury may reduce an age-dependent increase in the number of myelinated axons that would otherwise occur (Leenen et al., 1989).

\section{Can retrograde atrophy be detected by analyses of the medullary pyramid?}

Although there was no evidence of axon degeneration in the pyramid after SCI, previous studies have reported atrophy and shrinkage of the cell bodies of cortical motoneurons (Holmes and May, 1909; Lassek, 1942; Kalil and Schneider, 1975; Barron and Dentinger, 1979; Ganchrow and Bernstein, 1985; Ramirez and Kalil, 1985; Barron et al., 1988; Merline and Kalil, 1990; Tseng and Prince, 1996). Accordingly, it was of interest to determine whether it was possible to detect atrophy of CST axons in the pyramid. To assess this, we measured the cross-sectional area of a sample of CST axons (for the sampling strategy, see Fig. $8 \mathrm{~A}$ ). A particularly useful comparison is between the axons ipsilateral (unlesioned) and contralateral (lesioned) to a C5 hemisection injury because the unilateral injury provides an internal control. Cases that were completely unilateral $(n=2)$ for the C5 hemisection at 3 weeks were used for internal comparison of lesioned versus unlesioned axons. There was no difference in average axon diameter between the two sides 3 weeks after a cervical hemisection injury indicating no detectable atrophy (Fig. $8 B$ ). Indeed, there was not only no statistical difference; the means were almost identical.

Similar assessments revealed no differences in the crosssectional area of CST axons between uninjured young versus 
age-matched rats with T9 contusion injuries 3 weeks after contusion (Fig. 8C). Interestingly, however, axonal crosssectional area was less in rats that received T9 contusions 1 year previously compared with age-matched controls (Fig. 8C). The values from 1-year-old uninjured control rats were also significantly higher than the values from animals 3 weeks after hemisection. None of the other comparisons were statistically significant, however (Fig. 8C), including the trend for increased axon area in the old versus young uninjured rats-a phenomenon that has previously been reported for axons in dorsal roots (Rao and Krinke, 1983).

Together, these results reveal that there are no detectable changes in axon size during the time that cortical motoneurons exhibit retrograde atrophy after SCI, but SCI may affect age-related changes in axon size that otherwise occur.

Could spinal cord lesions lead to a selective death of unmyelinated axons and sparing of myelinated axons?

One possible explanation for the absence of degeneration of myelinated axons is that cortical motoneurons that die after spinal cord injury project via unmyelinated axons. This is highly unlikely in principle because cells that have been reported to exhibit caspase staining are large neurons in layer V. Nevertheless, to rule out this possibility, we assessed whether a significant number of CST axons (identified by BDA tracing) were unmyelinated. EM analyses after BDA tract-tracing injections into the sensorimotor cortex revealed that the vast majority of BDA-

labeled axons in the pyramid are myelinated (Fig. 9C). There were very few electron-dense processes that we conservatively classified as labeled unmyelinated axons because they appeared to contain neurofilaments and microtubules, but definitive identification of these as unmyelinated axons was difficult because the BDA labeling obscured the cytoplasm. Even including these questionable elements, the number of labeled unmyelinated axons was low (16.9 \pm 9.3 labeled myelinated axons/275 $\mu \mathrm{m}^{2}$ vs $0.7 \pm 1.1$ labeled unmyelinated axons $/ 275 \mu \mathrm{m}^{2}$ ).

\section{Discussion}

Given the long-standing controversy over whether there is retrograde cell death of the CST after SCI, the present study aimed to resolve this matter definitively in a novel way, by assessing CST axons in the medullary pyramid. Our results reveal that there is no degeneration of CST axons or axon loss in the medullary pyramid, even in situations that have previously been reported to cause extensive retrograde degeneration of cortical motoneurons (Hains et al., 2003). Thus, cortical motoneurons and their axons in the medullary pyramid survive the injury to the distal axon after various types of spinal cord injury. The important implication is that interventions to promote regeneration of the CST do not require separate manipulations to preserve the cells of origin in the cortex.

\section{B Pyramid Area Following C5 $\mathrm{Hx}$}

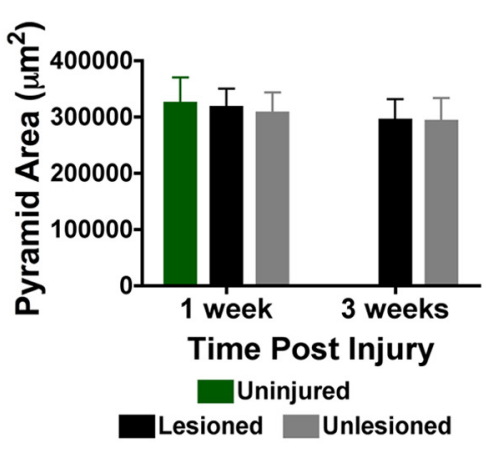

D Axon Counts (Total vs. Degenerating) Following $\mathrm{C} 5 \mathrm{Hx}$

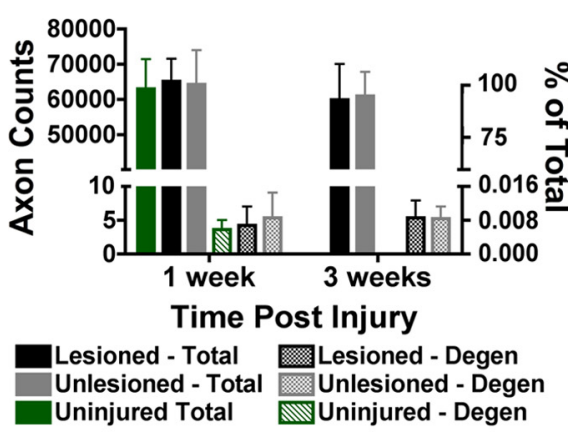

Figure 7. Effect of lesion extent on axon degeneration in the medullary pyramid. $A, C$, Lesions at $C 5$ were reconstructed by assessing horizontal sections through the lesion epicenter at $100 \mu \mathrm{m}$ intervals and representing these on schematic cross sections. I week $(\boldsymbol{A})$ and 3 week $(\boldsymbol{C})$ survival. $\boldsymbol{B}, \boldsymbol{D}$, Quantification of area, degenerating axons, and total axons in the medullary either the area of the pyramid $(\boldsymbol{B})$ or axon degeneration as measured by both total axons as well as axons displaying degenerative the spinal cord that were mostly affected across all the animals. The gray scale under each reconstruction depicts the variability in the lesion sizes, with darker regions representing co-incidence of lesion area, shown in $A$ and $C$. The darkest gray indicates the area damaged in all animals; the lightest indicates the area damaged in only one animal. Scale bar, $500 \mu \mathrm{m}$. Data are mean \pm SD.

It should be noted that we undertook this study with the expectation that retrograde degeneration did occur and that it would be possible to easily detect and rigorously quantify CST axon degeneration in the medullary pyramid. Thus, our results were as surprising to us as they are likely to be to the field.

\section{Assessment of CST axon degeneration}

Our assessment of CST axon degeneration in the medullary pyramid is based on the assumption that axons of neurons that die as a result of retrograde degeneration would exhibit degenerative changes similar to what are seen during Wallerian degeneration (condensation of the axonal cytoplasm, dark staining in toluidine blue-stained plastic sections, and eventually myelin fragmentation). There has been speculation about this issue after SCI in primates (Davison, 1937; Lassek, 1942), but no definitive evidence has been reported to our knowledge. Wallerian degeneration of an axon occurs after disconnection of the axon from its respective cell body (Waller, 1850) and has been reported extensively in the distal segment of the axon after injury (Ramon $y$ Cajal, 1928; Raff et al., 2002). Based on the rapid onset of degenerative changes in axons after damage to the cell body (Ranson, 1914; Dunkerley and Duncan, 1969; Beirowski et al., 2004; Coleman, 2005), time points of 1, 3, and 4 weeks after injury 
A Sampling Strategy in Medullary Pyramid for Assessment of Axon Atrophy

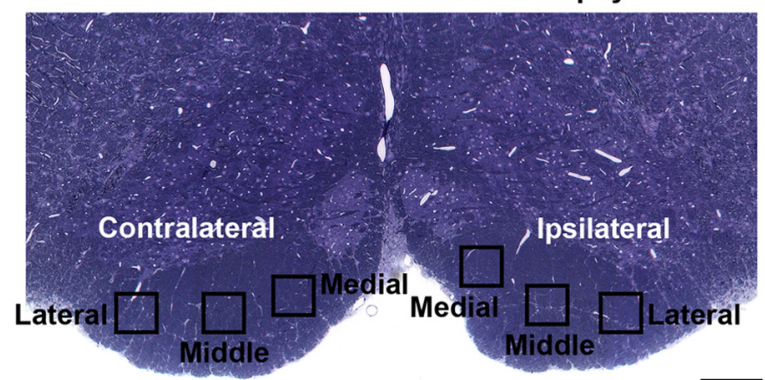

\section{B No Evidence of Atrophy in C5 Hx with Internal Control \\ C Decreased Age Related Axon Area Following SCl}
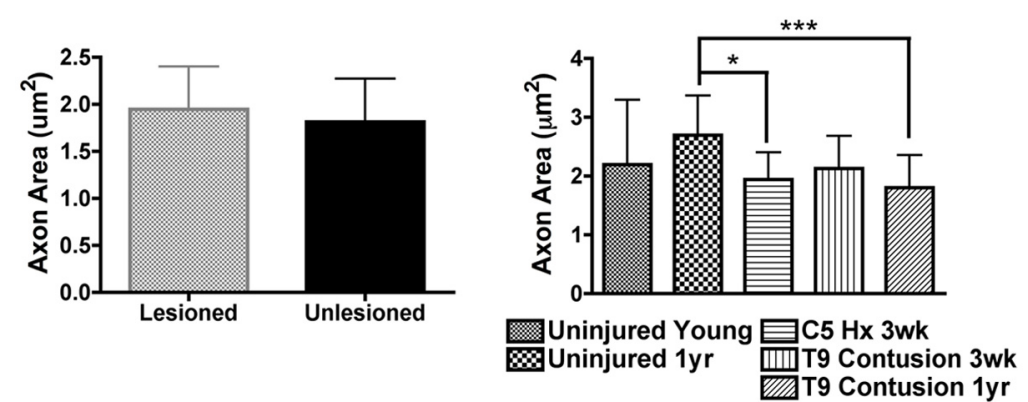

Figure 8. Assessment of axonal atrophy in the medullary pyramid. $\boldsymbol{A}$, Sampling strategy of axons in the pyramid, shown at low magnification in toluidine blue-stained plastic sections $(10 \times)$. Both sides of the pyramid were assessed at high magnification of $120 \times$ (sampling size illustrated in black boxes). Three sites per side were assessed, lateral, middle, and medial in the pyramid both for contralateral and ipsilateral sides. At each sampling site, three sites were used to measure axon area using fractionator sampling, and quantified using ImageJ. B, C, Quantification of axon area in the pyramids after $55 \mathrm{Hx}$ with 3 week survival, and T9 contusion with both 3 week and 1 year survival, compared with uninjured age-matched controls. $\boldsymbol{B}$, Lesioned (gray checkered bar) and unlesioned (black bar) pyramids within the same animal after a 5 Hx results in no significant difference between axon area between sides. C, Axon area is significantly decreased 3 weeks after a $\mathrm{C} 5 \mathrm{Hx}(p<0.05)$, and 1 year after a T9 contusion ( $p<$ 0.001 ), compared with the 1-year-old uninjured control; however, all other comparisons are not significantly different from each other. Scale bar, $200 \mu \mathrm{m}$. Data are mean \pm SD. ${ }^{*} p<0.05,{ }^{* * *} p<0.001$.

should be sufficient to detect degenerative changes in axons caused by retrograde degeneration of the cell body. Our control experiments involving aspiration lesions of the cortex reveal that CST axons exhibit Wallerian degeneration that is easily detectable by the techniques used here, confirming what has been seen in previous studies (Ranson, 1914; Dunkerley and Duncan, 1969).

Axon degeneration has also been reported after nontraumatic death of neurons in the CNS, for example, after death of neurons in the medial habenula caused by systemic administration of nicotine, causing axon degeneration in the fasciculus retroflexus (Carlson et al., 2000, 2001). Similarly, excitotoxic death of neurons is followed by Wallerian degeneration of their axons, which is easily detectable using selective silver impregnation techniques (Schmued et al., 1995; Schauwecker et al., 2000; Schauwecker, 2002; Benkovic et al., 2004), the methods of which have been described previously (Fink and Heimer, 1967; Beltramino et al., 1993; de Olmos et al., 1994). It remains possible, however, that retrograde degeneration of a neuronal cell body would lead to degeneration of the axon in a way that would not be detectable by the techniques used here (i.e., something other than condensation and dark staining of the axonal cytoplasm). It is also possible that the axons of cell bodies undergoing apoptosis may have an independent mechanism of degeneration than the cell body, which was suggested after cell culture studies in mice expressing the "slow Wallerian degeneration" $\left(\mathrm{Wld}^{\mathrm{s}}\right.$ ) allele (Deckwerth and Johnson, 1994).

\section{Counts of healthy axons}

If retrograde degeneration of cortical motoneurons occurs in a way that does not lead to detectable degenerative changes in the axons, counts of healthy axons would still reveal loss of axons in the pyramid proportional to the number of cortical motoneurons that died. For example, Hains et al. (2003) reported a loss of 20\% of the CST cell bodies 1 week after a T9 DF SCI. Our counting technique would certainly detect this because counts reveal significant axon loss in the medullary pyramid after either complete aspiration of the sensorimotor cortex, or after the more limited damage caused by injections for tract tracing. Previous published counts of healthy axons in the pyramid have ranged from 73,000 when assessed by light microscopy (Lassek and Rasmussen, 1940), up to $103,000-150,000$ when assessed by electron-microscopic counts (Leenen et al., 1985, 1989; Gorgels, 1990). Our counts are consistent with the previous counts based on light microscopy.

It is noteworthy that, after complete aspiration of the sensorimotor cortex, which destroys all of the cells of origin of the CST that are retrogradely labeled by Fluorogold injections into the spinal cord (Strong et al., 2009), approximately onehalf of the axons in the medullary pyramid survived. Previous studies have also noted that a significant proportion of the axons in the pyramid remained healthy after large cortical ablations in rats (Ranson, 1914; Dunkerley and Duncan, 1969). This indicates that axons in the medullary pyramid originate in part from areas other than the sensorimotor cortex. Early studies speculated that these surviving axons might be originating from a subcortical region (Ranson, 1914) such as the lentiform nucleus (Swank, 1936). More recent studies suggest a cortical origin, however, based on retrograde labeling of neurons in posterior parietal, rostral occipital, anterior cingulate, and prefrontal cortex after injections of the retrograde tracer HRP at C5/6 in rats (Miller, 1987).

One other body of literature deserves note: Brodal and Walberg (1952) do report the presence of degenerating axons in the medullary pyramid after spinal cord injuries in cats based on silver staining (the Glees method). Their speculation was that the axons might be from ascending sensory systems. This issue was further explored, but not resolved in later studies (Brodal and Walberg, 1982), which also contains an excellent discussion of the evidence for indirect Wallerian degeneration. Although we did not detect evidence of degenerative changes in axons after spinal cord injury, it is possible that a small number of axons do undergo some sort of degenerative change that would be detected using selective silver impregnation.

Could new axons grow into the medullary pyramid to replace ones that degenerate?

One possible alternative explanation for the lack of any detectable loss of myelinated axons in the medullary pyramid is that new axons grow in at the same time that others degenerate. In this 
regard, recent studies have revealed that, after C3/4 lateral hemisections, there are increased numbers of ipsilateral CST projections at cervical levels (Ghosh et al., 2009). Similarly, after T9 dorsal hemisections, there are increases in CST projections in segments rostral to the injury (Ghosh et al., 2010). These increases in CST axons could conceivably involve growth of new axons from the cortex, but the evidence is more consistent with local sprouting within the spinal cord. Also, given the lack of change in axon numbers, some axons would have to degenerate as others grow in, and we see no evidence for such degeneration. Finally, new axons that grew in would have to be myelinated to be counted in our assessments, and there was no evidence of new myelin (which would be evident as a thinner than normal myelin sheath). Taking everything together, the possibility of axon degeneration and compensatory new axon ingrowth into the pyramid seems highly unlikely.

\section{Could retrograde degeneration involve only a subset of cortical motoneurons} that project via unmyelinated axons?

The fact that there was no detectable loss of myelinated axons after SCI could be explained if the previously reported retrograde degeneration involved only a subset of cortical motoneurons whose axons were unmyelinated. This seems highly unlikely, because the retrograde degeneration reported by Hains et al. (2003) appeared to involve neurons of all sizes including the largest neurons in layer $\mathrm{V}$, whose axons are likely to be large and myelinated. Also, our data reveal very few labeled unmyelinated axons after BDA injections into the sensorimotor cortex.

\section{Is age-dependent myelination disrupted by SCI?}

Our data from uninjured controls reveal higher numbers of myelinated axons in older rats compared with young adults. This confirms previous studies showing an increase in myelinated axons in the pyramid between 2 and 14 months of age (Leenen et al., 1989). In contrast, the numbers of myelinated axons in rats with 1-year-old contusion injuries ( $\sim 15$ months of age) were not higher than in young adult rats $(\sim 3$ months of age). We speculate that spinal cord injuries may somehow impede the agedependent increase in myelination that would normally occur. Mechanisms underlying this reduction in myelination remain to be elucidated.

\section{Comparison of results in different rat strains}

Our study definitively establishes that cortical motoneurons do not die after SCI in Sprague Dawley rats, despite previous reports suggesting cortical motoneuron degeneration (Hains et al., 2003; Lee et al., 2004). Some other previous studies of retrograde changes in cortical motoneurons have involved different strains, however. For example, one study of cortical motoneurons after T8/9 injuries in Wistar rats reports a 30\% loss of cortical motoneurons, which was reduced by suppressing scar formation at the lesion site (Klapka et al., 2005). Another study involving locally bred isogenic female rats derived from Wistar rats reports a substantial decrease (93\%) in the number of neurons that were retrogradely labeled after tracer injections rostral to a lesion made 1 year previously (Feringa and Vahlsing, 1985). The decrease in labeling in this situation could be attributable to reduced uptake because of retraction of amputated axons at the level of the injury (Pallini et al., 1988), but it cannot be excluded that cortical motoneurons do die after SCI in strains or species other than Sprague Dawley rats.

\section{Implications for regenerative therapy}

If there were in fact a 20\% loss of the CST after the first week after injury, this would suggest a critical need for therapeutic intervention in a very narrow time window for there to be any hope of promoting regeneration of damaged CST axons in the spinal cord. Our results provide conclusive evidence, however, that there is minimal if any degeneration of the CST after various types of spinal cord injury. These data eliminate from consideration what was previously thought to be an important therapeutic target. In our view, eliminating a potential therapeutic target is of high significance because it allows research efforts and resources to be directed in other, more fruitful directions.

\section{References}

Anderson KD, Gunawan A, Steward O (2005) Quantitative assessment of forelimb motor function after cervical spinal cord injury in rats: relationship to the corticospinal tract. Exp Neurol 194:161-174.

Barron KD, Dentinger MP (1979) Cytologic observations on axotomized feline Betz cells. 1. Qualitative electron microscopic findings. J Neuropathol Exp Neurol 38:128-151. 
Barron KD, Dentinger MP, Popp AJ, Mankes R (1988) Neurons of layer Vb of rat sensorimotor cortex atrophy but do not die after thoracic cord transection. J Neuropathol Exp Neurol 47:62-74.

Basso DM, Beattie MS, Bresnahan JC (1995) A sensitive and reliable locomotor rating scale for open field testing in rats. J Neurotrauma 12:1-21.

Beirowski B, Berek L, Adalbert R, Wagner D, Grumme DS, Addicks K, Ribchester RR, Coleman MP (2004) Quantitative and qualitative analysis of Wallerian degeneration using restricted axonal labelling in YFP-H mice. J Neurosci Methods 134:23-35.

Beltramino CA, de Olmos JS, Gallyas F, Heimer L, Záborszky L (1993) Silver staining as a tool for neurotoxic assessment. NIDA Res Monogr 136:101126; discussion 126-132.

Benkovic SA, O'Callaghan JP, Miller DB (2004) Sensitive indicators of injury reveal hippocampal damage in $\mathrm{C} 57 \mathrm{BL} / 6 \mathrm{~J}$ mice treated with kainic acid in the absence of tonic-clonic seizures. Brain Res 1024:59-76.

Beresford WA (1965) A discussion on retrograde changes in nerve fibres. Prog Brain Res 14:33-56.

Bodian D (1946) Spinal projections of brain-stem in rhesus monkey, deduced from retrograde chromatolysis. Anat Rec 94:512-513.

Bonatz H, Röhrig S, Mestres P, Meyer M, Giehl KM (2000) An axotomy model for the induction of death of rat and mouse corticospinal neurons in vivo. J Neurosci Methods 100:105-115.

Bresnahan JC, Beattie MS, Stokes BT, Conway KM (1991) Threedimensional computer-assisted analysis of graded contusion lesions in the spinal cord of the rat. J Neurotrauma 8:91-101.

Brodal A, Walberg F (1952) Ascending fibers in pyramidal tract of cat. AMA Arch Neurol Psychiatry 68:755-775.

Brodal A, Walberg F (1982) A re-evaluation of the question of ascending fibers in the pyramidal tract. Brain Res 232:271-281.

Carlson J, Armstrong B, Switzer RC 3rd, Ellison G (2000) Selective neurotoxic effects of nicotine on axons in fasciculus retroflexus further support evidence that this a weak link in brain across multiple drugs of abuse. Neuropharmacology 39:2792-2798.

Carlson J, Noguchi K, Ellison G (2001) Nicotine produces selective degeneration in the medial habenula and fasciculus retroflexus. Brain Res 906:127-134.

Coleman M (2005) Axon degeneration mechanisms: commonality amid diversity. Nat Rev Neurosci 6:889-898.

Crawley AP, Jurkiewicz MT, Yim A, Heyn S, Verrier MC, Fehlings MG, Mikulis DJ (2004) Absence of localized grey matter volume changes in the motor cortex following spinal cord injury. Brain Res 1028:19-25.

Davison C (1937) Syndrome of the anterior spinal artery of the medulla. AMA Arch Neurol Psychiatry 37:91-107.

Deckwerth TL, Johnson EM Jr (1994) Neurites can remain viable after destruction of the neuronal soma by programmed cell death (apoptosis). Dev Biol 165:63-72.

de Olmos JS, Beltramino CA, de Olmos de Lorenzo S (1994) Use of an amino-cupric-silver technique for the detection of early and semiacute neuronal degeneration caused by neurotoxicants, hypoxia, and physical trauma. Neurotoxicol Teratol 16:545-561.

Dunkerley GB, Duncan D (1969) A light and electron microscopic study of the normal and the degenerating corticospinal tract in the rat. J Comp Neurol 137:155-183.

Feringa ER, Vahlsing HL (1985) Labeled corticospinal neurons one year after spinal cord transection. Neurosci Lett 58:283-286.

Fink RP, Heimer L (1967) Two methods for selective silver impregnation of degenerating axons and their synaptic endings in the central nervous system. Brain Res 4:369-374.

FitzGerald MJT, Gruener G, Mtui E (2007) Clinical neuroanatomy and neuroscience, Ed 5. Philadelphia: Elsevier Saunders.

Ganchrow D, Bernstein JJ (1985) Thoracic dorsal funicular lesions affect the bouton patterns on, and diameters of, layer VB pyramidal cell somata in rat hindlimb cortex. J Neurosci Res 14:71-81.

Ghosh A, Sydekum E, Haiss F, Peduzzi S, Zörner B, Schneider R, Baltes C, Rudin M, Weber B, Schwab ME (2009) Functional and anatomical reorganization of the sensory-motor cortex after incomplete spinal cord injury in adult rats. J Neurosci 29:12210-12219.

Ghosh A, Haiss F, Sydekum E, Schneider R, Gullo M, Wyss MT, Mueggler T, Baltes C, Rudin M, Weber B, Schwab ME (2010) Rewiring of hindlimb corticospinal neurons after spinal cord injury. Nat Neurosci 13:97-104.

Giehl KM, Tetzlaff W (1996) BDNF and NT-3, but not NGF, prevent axotomy-induced death of rat corticospinal neurons in vivo. Eur J Neurosci 8:1167-1175.

Gold BG, Griffin JW, Price DL (1992) Somatofugal axonal atrophy precedes development of axonal degeneration in acrylamide neuropathy. Arch Toxicol 66:57-66.

Gorgels TG (1990) A quantitative analysis of axon outgrowth, axon loss, and myelination in the rat pyramidal tract. Brain Res Dev Brain Res 54:51-61.

Griffin JW, George EB, Hsieh ST, Glass JD (1995) Axonal degeneration and disorders of the axonal cytoskeleton. New York, Oxford: Oxford UP.

Hains BC, Black JA, Waxman SG (2003) Primary cortical motor neurons undergo apoptosis after axotomizing spinal cord injury. J Comp Neurol 462:328-341.

Hammond EN, Tetzlaff W, Mestres P, Giehl KM (1999) BDNF, but not NT-3, promotes long-term survival of axotomized adult rat corticospinal neurons in vivo. Neuroreport 10:2671-2675.

Holmes G, May WP (1909) On the exact origin of the pyramidal tract in man and other mammals. Brain 32:1-43.

Kalil K, Schneider GE (1975) Retrograde cortical and axonal changes following lesions of the pyramidal tract. Brain Res 89:15-27.

Klapka N, Hermanns S, Straten G, Masanneck C, Duis S, Hamers FP, Müller D, Zuschratter W, Müller HW (2005) Suppression of fibrous scarring in spinal cord injury of rat promotes long-distance regeneration of corticospinal tract axons, rescue of primary motoneurons in somatosensory cortex and significant functional recovery. Eur J Neurosci 22:3047-3058.

Kost SA, Oblinger MM (1993) Immature corticospinal neurons respond to axotomy with changes in tubulin gene expression. Brain Res Bull 30:469-475.

Kost-Mikucki SA, Oblinger MM (1991) Changes in glial fibrillary acidic protein mRNA expression after corticospinal axotomy in the adult hamster. J Neurosci Res 28:182-191.

Kuypers HGJM (1981) Anatomy of the descending pathways. Baltimore: Williams and Wilkins.

Lassek AM (1942) The pyramidal tract: a study of retrograde degeneration in the monkey. Arch Neurol Psychiatr (Chicago) 48:561-567.

Lassek AM, Rasmussen GL (1940) A comparative fiber and numerical analysis of the pyramidal tract. J Comp Neurol 72:417-428.

Lee BH, Lee KH, Kim UJ, Yoon DH, Sohn JH, Choi SS, Yi IG, Park YG (2004) Injury in the spinal cord may produce cell death in the brain. Brain Res 1020:37-44.

Leenen LP, Meek J, Posthuma PR, Nieuwenhuys R (1985) A detailed morphometrical analysis of the pyramidal tract of the rat. Brain Res 359:65-80.

Leenen LP, Meek J, Posthuma PR, Nieuwenuys R (1989) Differences in the fiber composition of the pyramidal tract in two- and 14-month-old rats. Neuroscience 28:635-643.

Liu CN (1955) Time pattern in retrograde degeneration after trauma of central nervous system of mammals. In: Regeneration in the central nervous system (Windle WF, ed), pp 84-93. Springfield, IL: Thomas.

Mason MR, Lieberman AR, Anderson PN (2003) Corticospinal neurons up-regulate a range of growth-associated genes following intracortical, but not spinal, axotomy. Eur J Neurosci 18:789-802.

Merline M, Kalil K (1990) Cell death of corticospinal neurons is induced by axotomy before but not after innervation of spinal targets. J Comp Neurol 296:506-516.

Mikucki SA, Oblinger MM (1991) Corticospinal neurons exhibit a novel pattern of cytoskeletal gene expression after injury. J Neurosci Res 30:213-225.

Miller MW (1987) The origin of corticospinal projection neurons in rat. Exp Brain Res 67:339-351.

Neumann S, Woolf CJ (1999) Regeneration of dorsal column fibers into and beyond the lesion site following adult spinal cord injury. Neuron 23:83-91.

Nissl F (1892) Ueber die Veranderungen der Ganglienzellen am Facialiskern des Kaninchens nach Ausreissung der Nerven. Allgen Z Psychiatr 48:197-198.

Pallini R, Fernandez E, Sbriccoli A (1988) Retrograde degeneration of corticospinal axons following transection of the spinal cord in rats. A quantitative study with anterogradely transported horseradish peroxidase. J Neurosurg 68:124-128.

Piecharka DM, Kleim JA, Whishaw IQ (2005) Limits on recovery in the corticospinal tract of the rat: partial lesions impair skilled reaching and 
the topographic representation of the forelimb in motor cortex. Brain Res Bull 66:203-211.

Raff MC, Whitmore AV, Finn JT (2002) Axonal self-destruction and neurodegeneration. Science 296:868-871.

Ramirez LF, Kalil K (1985) Critical stages for growth in the development of cortical neurons. J Comp Neurol 237:506-518.

Ramon y Cajal S (1928) Cajal's degeneration and regeneration of the nervous system. New York, Oxford: Oxford UP.

Ranson SW (1914) A note on the degeneration of the fasciculus cerebrospinalis in the albino rat. J Comp Neurol 24:503-507.

Rao RS, Krinke G (1983) Changes with age in the number and size of myelinated axons in the rat L4 dorsal spinal root. Acta Anat (Basel) 117: 187-192.

Reh T, Kalil K (1981) Development of the pyramidal tract in the hamster. I. A light microscopic study. J Comp Neurol 200:55-67.

Rosenbluth (1995) Pathology of demylinated and dysmyelinated axons. New York, Oxford: Oxford UP.

Schauwecker PE (2002) Modulation of cell death by mouse genotype: differential vulnerability to excitatory amino acid-induced lesions. Exp Neurol 178:219-235.

Schauwecker PE, Ramirez JJ, Steward O (2000) Genetic dissection of the signals that induce synaptic reorganization. Exp Neurol 161:139-152.

Schmidlin E, Wannier T, Bloch J, Rouiller EM (2004) Progressive plastic changes in the hand representation of the primary motor cortex parallel incomplete recovery from a unilateral section of the corticospinal tract at cervical level in monkeys. Brain Res 1017:172-183.

Schmidlin E, Wannier T, Bloch J, Belhaj-Saif A, Wyss AF, Rouiller EM (2005) Reduction of the hand representation in the ipsilateral primary motor cortex following unilateral section of the corticospinal tract at cervical level in monkeys. BMC Neurosci 6:56.

Schmued LC, Scallet AC, Slikker W Jr (1995) Domoic acid-induced neuronal degeneration in the primate forebrain revealed by degeneration specific histochemistry. Brain Res 695:64-70.

Strong MK, Blanco JE, Anderson KD, Lewandowski G, Steward O (2009) An investigation of the cortical control of forepaw gripping after cervical hemisection injuries in rats. Exp Neurol 217:96-107.

Swank RL (1936) The pyramidal tract. An experimental study of the corticospinal tract and other components in the rabbit. AMA Arch Neurol Psychiatry 36:530-541.

Thuret S, Moon LD, Gage FH (2006) Therapeutic interventions after spinal cord injury. Nat Rev Neurosci 7:628-643.

Tseng GF, Prince DA (1996) Structural and functional alterations in rat corticospinal neurons after axotomy. J Neurophysiol 75:248-267.

Van Gehuchten A (1903) La degenerescence dite retrograde ou degenerescence wallerienne indirecte. Nevraxe 5:1-108.

Waller A (1850) Experiments on the section of the glossopharyngeal and hypoglossal nerves of the frog, and observations of the alterations produced thereby in the structure of their primitive fibres. Philos Trans R Soc London 140:423-429.

Wannier T, Schmidlin E, Bloch J, Rouiller EM (2005) A unilateral section of the corticospinal tract at cervical level in primate does not lead to measurable cell loss in motor cortex. J Neurotrauma 22:703-717.

Wiesendanger M (1981) The pyramidal tract. Its structure and function. New York: Plenum.

Zimmerman EA, Chambers WW, Liu CN (1964) An experimental study of the anatomical organization of the cortico-bulbar system in the albino rat. J Comp Neurol 123:301-323. 